\title{
Patterns of plasma cobalamins in control subjects and in cases of vitamin $B_{12}$ deficiency
}

\author{
J. C. LINNELL, HEATHER M. MACKENZIE, J. WILSON, \\ AND D. M. MATTHEWS
}

\begin{abstract}
From the Department of Chemical Pathology, Westminster Medical School, London, and the Medical Research Council Clinical Genetics Research Unit, Institute of Neurology, London
\end{abstract}

SYNOPSIS A method for thin-layer chromatography and bioautography of plasma cobalamins is described. It requires only $5 \mathrm{ml}$ of blood.

Results are reported in 35 healthy people and hospital controls, and in 14 cases of untreated pernicious anaemia. In the control group there were two major components, methylcobalamin and a component which is probably a mixture of hydroxocobalamin and deoxyadenosyl cobalamin. Many cases of untreated pernicious anaemia showed a characteristic pattern in which methylcobalamin was reduced in relation to hydroxocobalamin. This pattern was also seen in one vegan who was taking a diet without $\mathrm{B}_{12}$ supplement. A minority of subjects showed traces of cyanocobalamin; this compound was not confined to smokers.

The significance of the results and possible diagnostic utility of the technique are discussed.

For some years after the isolation of vitamin $B_{12}$ as cyanocobalamin in 1948, it was assumed that the 'serum vitamin $\mathbf{B}_{12}$ ', estimated by the usual microbiological methods, was either cyanocobalamin or a mixture of cyanocobalamin and hydroxocobalamin (Wokes and Picard, 1955; Matthews, 1961) though the demonstration of a coenzyme form of vitamin $B_{12}$ in human and animal liver (Toohey and Barker, 1961) raised the possibility that this might also occur in the blood. In 1963, Lindstrand and Ståhlberg showed for the first time, by chromatography and bioautography, that human plasma contained several different microbiologically active cobalamins. These were provisionally identified as methylcobalamin, hydroxocobalamin, cyanocobalamin, and deoxyadenosyl cobalamin (Lindstrand and Ståhlberg, 1963; Lindstrand, 1964; Ståhlberg, 1964). This and more recent work has identified the major component as methylcobalamin with a high degree of certainty, but suggests that the component originally thought to be deoxyadenosyl cobalamin (coenzyme $\mathrm{B}_{12}$ ) may in fact be largely or entirely hydroxocobalamin (Ståhlberg, 1967).

It is clearly desirable to know as much as possible about the normal proportions of the compounds making up 'serum $\mathbf{B}_{12}$ ', and how they vary in disease. It is quite possible that a normal figure for total

Received for publication 4 March 1969. serum $B_{12}$ might conceal some imbalance in individual plasma cobalamins. It might also mask a deficiency of physiologically active cobalamins, owing to their conversion to cyanocobalamin, which is without coenzyme function and is readily excreteda state of affairs which might lead to haematological and neurological disorders. The hypothesis that an excessive load of cyanide, which may result from heavy smoking, the consumption of cassava, certain infections, and possibly from an error in cyanide metabolism, may inactivate vitamin $B_{12}$ in this way is now suggested by more than one line of evidence (Wilson and Matthews, 1966; Linnell, Smith, Smith, Wilson, and Matthews, 1968). It has recently been referred to by Lester Smith (1968) as the hypothesis of 'trapped $B_{12}$ '.

The ability to determine patterns of plasma cobalamins reasonably readily might lead to significant advances in understanding of $\mathrm{B}_{12}$ metabolism and in haematological and neurological diagnosis. The reason why such determinations have not become generally possible is basically technical, and the difficulties are contributed to by the very large volume of blood (at least $40 \mathrm{ml}$ ) required for the method of Lindstrand and Ståhlberg. This paper describes a chromatographic and bioautographic technique using thin-layer chromatography and requiring only $5 \mathrm{ml}$ of blood. While we do not claim that the method is easy, it is more convenient than 
that of Lindstrand and Stahlberg (1963) and enables up to 20 analyses to be carried out per week. The results obtained in normal subjects, hospital control patients, and patients with $B_{12}$ deficiency are reported.

\section{MATERIALS AND METHODS}

Control blood samples were taken from healthy laboratory staff and hospital inpatients without anaemia.

Total plasma $\mathbf{B}_{12}$ was estimated by radioisotopic assay (Matthews, Gunasegaram, and Linnell, 1967).

CHROMATOGRAPHIC PROCEDURE Venous blood (5 to $10 \mathrm{ml}$ ) is taken in a foil-wrapped heparinized container. Plasma is separated within $30 \mathrm{~min}$ by red darkroom light, and stored deep-frozen until analysis.

The procedures to be described are carried out by darkroom light or in total darkness when possible.

Plasma $(2 \mathrm{ml})$ is mixed with absolute ethanol $(8 \mathrm{ml})$ and heated at $80^{\circ} \mathrm{C}$ for $20 \mathrm{~min}$, cooled in ice, and filtered on a sintered glass filter (grade 3 ). The protein precipitate is washed with cold ethanol and the filtrate transferred to a rotary evaporator (Buchi Rotavapor-R). The temperature of the water bath is slowly raised from $25^{\circ} \mathrm{C}$ to $40^{\circ} \mathrm{C}$ as ethanol distils. The aqueous residue is thrice washed with ether and evaporated to dryness. The residue is redissolved in water $(2 \mathrm{ml})$ and phenol containing $25 \%$ water $(8 \mathrm{ml})$ and placed in a stoppered tube. The aqueous phase is removed and the lower phenol layer washed with water (three $2 \mathrm{ml}$ aliquots). Acetone $(7 \mathrm{ml})$ and ether $(21 \mathrm{ml})$ are added to the phenol and shaken with water $(1 \mathrm{ml})$ to extract the cobalamins. The aqueous extract is then washed with ether and desalted for $10 \mathrm{~min}$ in an ion-exchange cell (Baird and Tatlock chromatographic desalting apparatus). Finally, the desalted extract is evaporated to dryness.

Thin-layer plates are prepared by mixing powdered cellulose (Whatman Chromedia CC 41) (15 g) with silica gel (Merck silica gel G) $(5 \mathrm{~g})$ and slurried in water (45 $\mathrm{ml}$ ). A layer $0.25 \mathrm{~mm}$ thick is then applied to glass plates and the adsorbent dried in the oven at $100^{\circ} \mathrm{C}$ for 30 minutes.

The plasma extract is redissolved in water $(25 \mu 1)$ and applied to a thin-layer plate ( 1 and $4 \mu 1$ loadings normally, 2 and $6 \mu 1$ if plasma $B_{12}$ is low) together with markers in aqueous solution (hydroxocobalamin, cyanocobalamin, deoxyadenosyl cobalamin, and methylcobalamin: $50 \mu \mu \mathrm{g}$ of each in $1 \mu 1$ ). The chromatogram is developed for three hours at $21^{\circ} \mathrm{C}$ in secbutanol/0.880 ammonia/water $(75: 2: 25)$, removed from the solvent and dried. Subsequent steps are carried out by daylight.

BIOAUTOGRAPHIC PROCEDURE A $20 \mathrm{~cm}$ square of Whatman no. 1 filter paper is laid on the previously prepared bioautography plate and a $20 \mathrm{~cm}$ square of agar cut out. Agar and paper are lifted together and placed on the chromatogram, agar side uppermost. Air bubbles are displaced and the 'sandwich' is incubated at $37^{\circ} \mathrm{C}$ overnight.

A trace amount of cobalamin promotes growth of an
E. coli mutant, which is indicated by conversion of $\frac{0}{a}$ tetrazolium salt to a coloured form. Red zones appea on the bioautograph plate, in positions corresponding to the zones separated by chromatography.

Preparation of seeded agar for bioautography standard blood agar plate is inoculated with $E$. coti NCIB-9270 and incubated at $37^{\circ} \mathrm{C}$ overnight. The growt is subcultured in peptone water $(2 \mathrm{ml})$, incubated for sis to eight hours, and used as the inoculum.

The agar medium is made up in two parts and store separately:

Agar Davis New Zealand Agar (3 g) in water (100 m\$) is autoclaved and stored at room temperature.

Medium Dipotassium hydrogen phosphate . . Potassium dihydrogen phosphate .. Sodium citrate .. .. . . Magnesium sulphate heptahydrate Ammonium sulphate .. .. Sodium chloride $\ldots$ $\begin{array}{llll}. . & . . & . & 0.018\end{array}$ Distilled water to $\quad . . \quad \ldots \quad 100 \mathrm{ml}$

The medium is filtered, the $p \mathrm{H}$ adjusted to $7 \cdot 0$, them autoclaved and stored at room temperature.

The agar is melted on a steam bath while glucost $(0.6 \mathrm{~g})$ and $2,3,5$ triphenyltetrazolium chloride $(0.03 \mathrm{~g}$ are dissolved in the medium. A bioautography plate (constructed from two plates of glass $25 \mathrm{~cm}$ square separated by a Perspex frame $1 \mathrm{~cm}$ thick) is cleaned be wiping with ethanol and flaming. The melted agar is poured into the medium, mixed, and cooled to $43^{\circ} \mathrm{C}$ The inoculum is added and the seeded agar poured ont $\$$ the bioautography plate set on a level bench.

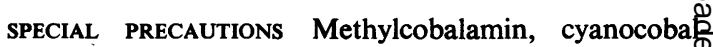
amin, and deoxyadenosyl cobalamin are all converted to hydroxocobalamin on exposure to light. For this reason, samples must be protected from light both befores and during analysis to prevent alteration of the chromator graphic pattern. Syringes and blood containers ares wrapped in aluminium foil, while all steps in the extracs. tion and chromatography procedures are carried ous either in darkness or by the light of a darkroom lamig (Paterson Safelight).

To prevent contamination of bioautographs, all con centrated cobalamin solutions are prepared in a labos atory remote from that used for the extraction of plasma and chromatography.

RECORDING RESULTS A 'plus' system has been used to record chromatographic results, each growth area being assigned from 0 to $3+$ signs. Scoring is carried of according to the area and intensity of each spot ip relation to the total growth on the chromatogram, so that the score given to an individual spot is related to the proportion of the total it represents, not to absolute amount. Where possible, chromatograms were scored 'blind', without knowledge of whether the sample wag normal or otherwise.

\section{RESULTS AND DISCUSSION}

Though cobalamins are readily separated by papeip or thin-layer chromatography, appearing wheg 


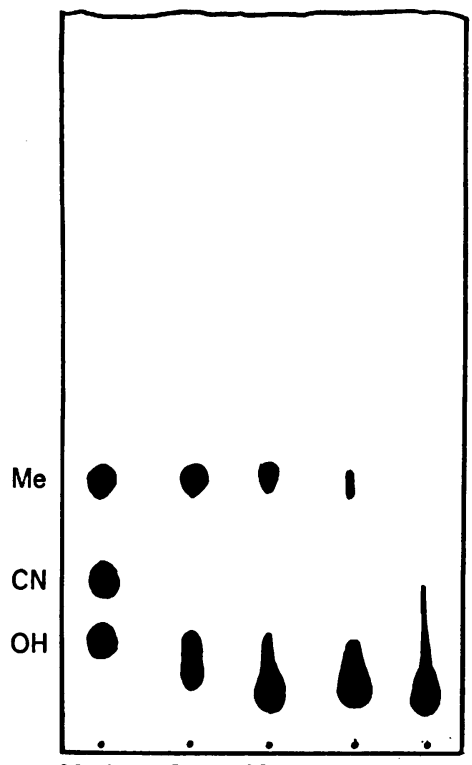

Markers $2 \mathrm{sec} .20 \mathrm{sec} .2 \mathrm{~min} .20 \mathrm{~min}$. FIG. 1.

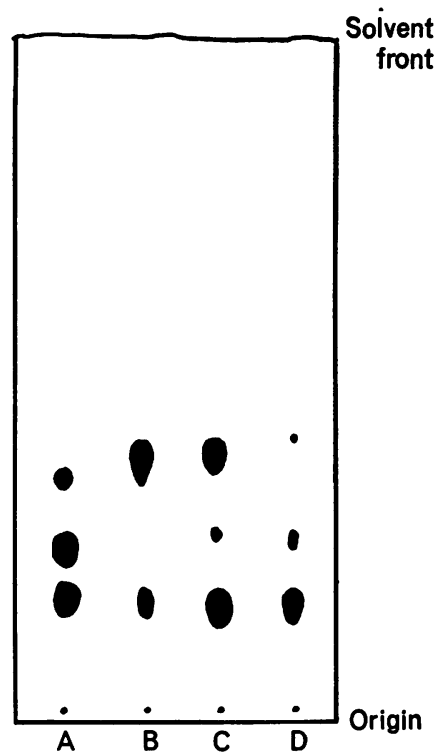

FIG. 1. Photolysis of methylcobalamin in normal plasma exposed to daylight for 20 minutes. At the end of the period all the methylcobalamin has been converted to hydroxocobalamin.

FIG. 2. Results of chromatography and bioautography of plasma cobalamins. A Markers (methylcobalamin, cyanocobalamin and hydroxocobalamin); B normal subject; C normal subject, showing trace of cyanocobalamin; D case of untreated pernicious anaemia, showing great reduction in methylcobalamin and a small amount of cyanocobalamin

FIG. 2.

present in high concentrations as red or orange spots, their concentrations in plasma are so low that the resulting spots can only be made visible by the technique of bioautography. The present method has certain advantages over the paper chromatographic technique (Lindstrand and Ståhlberg, 1963; Ståhlberg, 1967) apart from the much smaller quantity of blood required. It is simpler to manipulate thin-layer plates, and layer them evenly with bioautographic media than to deal with large $(22 \times 18$ in.) paper sheets. The use of staining to make growth zones more clearly visible makes it easy to identify very small amounts, and to make a photographic record. The definition of growth zones is good provided loading is not excessively heavy, and 'streaking' is minimal or absent. The important cyanocobalamin spot is well separated and can be clearly seen without prior removal of hydroxocobalamin, which was necessary with the paper technique (Lindstrand, Wilson, and Matthews, 1966).

Though the present chromatographic system separates methylcobalamin, cyanocobalamin, hydroxocobalamin, and deoxyadenosyl cobalamin satisfactorily from aqueous solutions containing high concentrations of these compounds, adequate separation of deoxyadenosyl cobalamin and hydroxocobalamin is not usually achieved when these two compounds are added to plasma in low concentrations $(1,000 \mu \mu \mathrm{g}$ per $\mathrm{ml}$ or less) both compounds tending to be represented by a single zone of growth.
The work of Ståhlberg (1967) suggests that there is little or no deoxyadenosyl cobalamin in plasma, in which case this growth area will normally represent hydroxocobalamin. We are not, however, satisfied that this is so, and for this reason the area is referred to below as 'hydroxocobalamin' in inverted commas.

The great importance of avoiding even short periods of exposure to light is illustrated in Figure 1. This shows that two minutes' exposure of plasma to daylight will convert nearly all its methylcobalamin, normally a major component, to hydroxocobalamin. Brief exposure of whole blood to light, on the other hand, has little effect.

The results obtained in healthy people and in hospital controls (Fig. 2, Tables I and III) were reasonably similar, and suggest that in the majority of cases, at least half the plasma $B_{12}$ is in the form of methylcobalamin. In about half the individuals in the pooled control group (normals and hospital patients) the methylcobalamin growth zone was approximately equal to that of 'hydroxocobalamin'. In about one third, the methylcobalamin zone was definitely predominant. In a minority, "hydroxocobalamin' was the predominant zone. A zone with the $\mathbf{R}_{\mathbf{F}}$ of cyanocobalamin appeared inconstantly, and when present was always small. The presence of this component was not confined to smokers. It tended to appear more frequently in them $(50 \%)$ than in non-smokers $(24 \%)$ but the difference was not statistically significant $\left(\chi^{2}=2 \cdot 56: P>0.083\right)$. 
TABLE I

RESULTS IN NORMAL SUBJECTS AND HOSPITAL CONTROLS

\begin{tabular}{|c|c|c|c|c|c|c|c|}
\hline & \multirow{2}{*}{ Age } & \multirow{2}{*}{ Sex } & \multirow{2}{*}{$\begin{array}{l}\text { Total } \\
\text { Plasma } B_{12} \\
(\mu \mu g / m l)\end{array}$} & \multirow{2}{*}{$\begin{array}{l}\text { Smoking } \\
\text { Habits } \\
\text { (cigarettes/day) }\end{array}$} & \multicolumn{3}{|c|}{ Plasma Cobalamins } \\
\hline & & & & & $O H-B_{12}$ & $C N-B_{12}$ & $M e-B_{12}$ \\
\hline \multicolumn{8}{|c|}{ Normal Subjects } \\
\hline 1 & 34 & $\mathbf{M}$ & 470 & Nil & $+t+$ & 0 & $++t$ \\
\hline 2 & 32 & $\mathbf{M}$ & 505 & $\mathrm{Nil}$ & $+t+$ & 0 & $t+t$ \\
\hline 3 & 22 & $\mathbf{F}$ & 380 & $\mathrm{Nil}$ & $+t+$ & 0 & $+t+$ \\
\hline 4 & 35 & $\mathbf{M}$ & 625 & $\mathrm{Nil}$ & +++ & 0 & ++ \\
\hline 5 & 21 & $\mathbf{F}$ & 250 & $\mathrm{Nil}$ & $+t+$ & \pm & $+t+$ \\
\hline 6 & 21 & $\mathbf{M}$ & 270 & $\mathrm{Nil}$ & $+t$ & + & $++t$ \\
\hline 7 & 25 & $\mathbf{F}$ & 450 & Nil & +4 & 0 & $4+\div$ \\
\hline 8 & 24 & $\mathbf{F}$ & 375 & $\mathrm{Nil}$ & $+i$ & 0 & +++ \\
\hline 9 & 27 & $\mathbf{M}$ & 345 & $\mathrm{Nil}$ & $+t$ & 0 & $\cdots++$ \\
\hline 10 & 30 & $\mathbf{M}$ & 415 & $\mathrm{Nil}$ & + & 0 & $++t$ \\
\hline 11 & 30 & $\mathrm{~F}$ & 375 & $\mathrm{Nil}$ & $+t$ & + & $++t$ \\
\hline 12 & 23 & $\mathbf{F}$ & - & Nil & +1 & 0 & +++ \\
\hline 13 & 26 & $\mathrm{~F}$ & 490 & $\mathrm{Nil}$ & $+t+$ & 0 & ++ \\
\hline 14 & 30 & $\mathbf{M}$ & 510 & Nil & $+t+$ & 0 & +++ \\
\hline 15 & 40 & $\mathbf{M}$ & 550 & $40 /$ day & ++4 & 0 & +++ \\
\hline 16 & 26 & $\mathbf{F}$ & 415 & $10 /$ day & $+\div$ & $\div$ & $\rightarrow \div$ \\
\hline 17 & 47 & $\mathbf{M}$ & 300 & $10 /$ day & ++4 & + & $+i$ \\
\hline 18 & 32 & $\mathbf{M}$ & - & $15 /$ day & +- & 0 & +++ \\
\hline 19 & 52 & $\mathbf{M}$ & 380 & $20 /$ day & +++ & + & + \\
\hline 20 & 26 & $\mathrm{~F}$ & 265 & $10 /$ day & +++ & 0 & $+t+t$ \\
\hline \multicolumn{8}{|c|}{ Hospital Controls } \\
\hline 1 & 48 & $\mathrm{~F}$ & - & Nil & $+t \cdot-t$ & 0 & $+t+$ \\
\hline 2 & 55 & $\mathbf{F}$ & 415 & $\mathrm{Nil}$ & $+\frac{1}{+}+$ & 0 & $++t$ \\
\hline 3 & 62 & $\mathbf{M}$ & 365 & Nil & $+i$ & 0 & $++t$ \\
\hline 4 & 78 & $\mathbf{M}$ & - & Nil & $+t+$ & + & ++ \\
\hline 5 & 60 & $\mathrm{~F}$ & 540 & $\mathrm{Nil}$ & $++i$ & + & $\div \quad \ldots$ \\
\hline 6 & 43 & $\mathbf{M}$ & 265 & Nil & $+t+$ & 0 & $+\ldots$ \\
\hline 7 & 40 & $F$ & 320 & Nil & +++ & 0 & +++ \\
\hline 8 & 63 & $\mathbf{M}$ & 575 & $10 /$ day & +++ & \pm & ++ \\
\hline 9 & 65 & F & 525 & 5/day & $t+t$ & 0 & $++t$ \\
\hline 10 & 42 & $\mathbf{M}$ & 430 & 5/day & $+t$ & 0 & $+t+$ \\
\hline 11 & 58 & $\mathbf{F}$ & 650 & $10 /$ day & $+t i$ & + & $+t+$ \\
\hline 12 & 44 & $\mathbf{M}$ & 455 & $15 /$ day & $++t$ & + & +++ \\
\hline 13 & 65 & $\mathbf{M}$ & 615 & 5/day & $+t+$ & \pm & $++t$ \\
\hline 14 & 67 & $\mathbf{M}$ & 530 & $50 /$ day & $+t+$ & 0 & 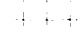 \\
\hline 15 & 59 & $\mathbf{M}$ & 285 & 5/day & + & 0 & ++1 \\
\hline
\end{tabular}

TABLE II

RESULTS IN PATIENIS WITH PERNICIOUS ANAEMIA AND IN VEGANS

Diagnosis

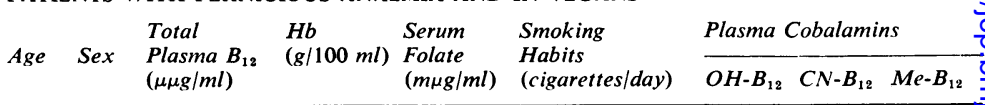

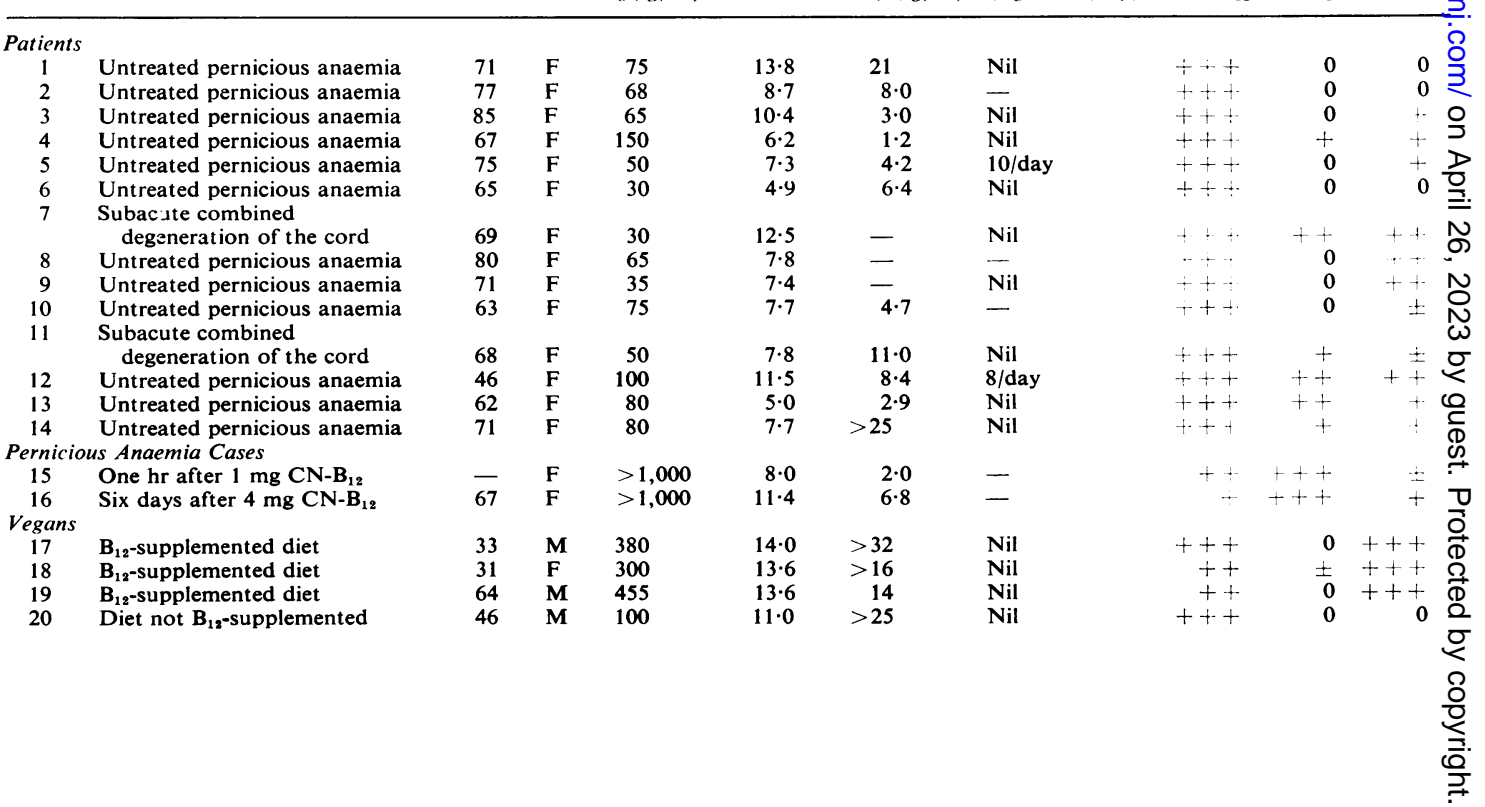


TABLE III

SUMMARY OF PATTERNS OF PLASMA COBALAMINS IN CONTROLS AND UNTREATED PERNICIOUS ANAEMIA

$\begin{array}{lc}\begin{array}{l}\text { Normals and } \\ \text { Hospital Controls } \\ (n=35)\end{array} & \begin{array}{l}\text { Untreated } \\ \text { Pernicious Anaemia } \\ (\%)\end{array} \\ (n=14)\end{array}$

\begin{tabular}{|c|c|c|}
\hline 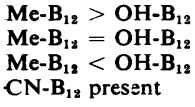 & $\begin{array}{rr}11 & 32 \\
17 & 48 \\
7 & 20 \\
12 & 34\end{array}$ & $\begin{array}{rr}0 & 0 \\
0 & 0 \\
14 & 100 \\
6 & 43\end{array}$ \\
\hline
\end{tabular}

Preliminary experiments on quantitation of the individual cobalamins, by elution of areas located with adjacent markers, gave the following results in three healthy people: subject 1 , total plasma $B_{12} 470$ $\mu \mu \mathrm{g}$ per $\mathrm{ml}$, methylcobalamin $57 \%$, 'hydroxocobalamin' $43 \%$; subject 2 , total $\mathrm{B}_{12} 620 \mu \mu \mathrm{g}$ per $\mathrm{ml}$, methylcobalamin $70 \%$, 'hydroxocobalamin' $30 \%$; subject 3, total $\mathrm{B}_{12} 350 \mu \mu \mathrm{g}$ per $\mathrm{ml}$, methylcobalamin $55 \%$, 'hydroxocobalamin' $45 \%$. No cyanocobalamin was present in any of these samples. The quantitative results were in general agreement with visual impressions. ${ }^{1}$

The pattern of plasma cobalamins in most cases of untreated pernicious anaemia (Fig. 2, Tables II and III) was quite abnormal, so much so that such cases could usually be picked out without any knowledge of the total plasma $B_{12}$ or reference to the absolute intensity of the zones of growth. In the majority of cases, the approximate equality between methylcobalamin and 'hydroxocobalamin' found in controls was lost, and 'hydroxocobalamin' was clearly the predominant component. In three of 14 cases $(21 \%)$ methylcobalamin was undetectable, and in most of the others present only in traces. Only four cases $(29 \%)$ had a pattern approximating to normal, and in all these, methylcobalamin was weaker than 'hydroxocobalamin'-a pattern which occurs in only a small proportion of control subjects. As in the control group, the cyanocobalamin growth zone was present in some cases (six, or $43 \%$ ) of untreated pernicious anaemia. In all of these cases, this zone was as strong as, or stronger than, that of methylcobalamin. It is interesting to compare two subjects in the 'low normal' range of total serum $B_{12}$. Subject 5 (Table I), a normal subject with a total serum $\mathbf{B}_{12}$ concentration of $250 \mu \mu \mathrm{g}$ per $\mathrm{ml}$, had roughly equal plasma concentrations of methylcobalamin and 'hydroxocobalamin'. Subject 4 (Table II), a case of pernicious anaemia with a total serum $\mathrm{B}_{12}$ concentration of $150 \mu \mu \mathrm{g}$ per ml, which falls at the lower limit of the normal range, had a clearly

\footnotetext{
${ }^{1}$ A larger series of quantitative values has recently been reported (Linnell, J. C., Mackenzie, H. M., and Matthews, D. M., 1969). J. clin. Path., 22, 506.
}

abnormal pattern of plasma cobalamins in which the proportion of methylcobalamin was unusually small. Thus estimation of individual plasma cobalamins might be useful in the differentiation of healthy subjects with relatively low serum vitamin $B_{12}$ from cases of incipient $B_{12}$ deficiency.

Table II shows two cases of pernicious anaemia after treatment with intramuscular cyanocobalamin. In patient 15 , one hour after treatment, the bulk of the plasma $B_{12}$ was in the form of cyanocobalamin, large amounts of this component being superimposed on the characteristic pernicious anaem:a pattern. In patient 16 , six days after treatment, much of the plasma $B_{12}$ still remained in this form. This is in keeping with other observations showing that conversion of cyanocobalamin to physiologically active forms is a relatively slow process (Reizenstein, 1967; Yagiri, 1967).

The pattern of plasma cobalamins in one untreated vegan, subject 20 , who had avoided $\mathbf{B}_{\mathbf{1 2}}$ supplementation of his diet but remained in reasonable health, was similar to that in untreated pernicious anaemia. In three vegans taking a $\mathrm{B}_{12}$-supplemented diet (nos. 17 to 19) the pattern was normal.

In some important respects, the present results agree with those of Lindstrand and Ståhlberg. They confirm the finding that in control subjects a major plasma $B_{12}$ component appears to be methylcobalamin, and that the second major component has an $\mathbf{R}_{\mathbf{F}}$ similar to that of hydroxocobalamin, though we are not yet certain whether or not this growth zone represents deoxyadenosyl cobalamin (coenzyme $\mathrm{B}_{12}$ ) in addition. The observation that at least half the plasma $B_{12}$ is usually in the form of methylcobalamin is borne out by the preliminary quantitative estimations. With respect to cyanocobalamin, the results differ. In their earliest report, Lindstrand and Ståhlberg (1963) found a component with the $R_{F}$ of cyanocobalamin in all eight people studied. In a second paper reporting results in 20 normal samples, the presence of this compound was not mentioned (Ståhlberg, 1964). In a more recent, and very detailed, report (Ståhlberg, 1967) plasma from 18 healthy people was examined and cyanocobalamin found in none; this paper refers to cyanocobalamin as an artefact with no metabolic function. On the other hand, we have found small amounts of a component with the $\mathbf{R}_{F}$ of cyanocobalamin in a substantial proportion ( $34 \%$ ) of 35 control subjects, including some non-smokers. It is known that cyanide participates in normal metabolic processes (Boxer and Rickards, 1952; Wilson and Matthews, 1966), and the cyanide pool is in equilibrium with thiocyanate, much of which, in non-smokers, is derived from foodstuffs. The formation of cyanocobalamin from other forms of vitamin $B_{12}$ may be 
part of one of the metabolic pathways normally available to the cyanide radicle.

- In pernicious anaemia, the two sets of results are totally different. Ståhlberg (1964) found that in untreated pernicious anaemia the plasma 'fourth factor' (methylcobalamin) tended to persist while the other component or components tended to disappear and were sometimes absent. A similar disturbance in the ratio of cobalamins was subsequently found in some samples of $B_{12}$-deficient liver, and led to the suggestion that in $B_{12}$ deficiency, $B_{12}$ might be blocked in the methyl form owing to a disturbance in demethylation (Ståhlberg, Radner, and Nordén, 1967). In contrast, we find that plasma methylcobalamin is severely reduced while the other major component 'hydroxocobalamin' tends to persist. We have at present no explanation for this discrepancy.

We are indebted to Dr L. Mervyn of Glaxo Laboratories for gifts of methylcobalamin, deoxyadenosyl cobalamin, and hydroxocobalamin, specially purified for use as chromatographic markers, and are also grateful to $\mathbf{M r}$
S. Vincent of Glaxo Laboratories for advice concern - 은 ing the bioautographic method and for the gift of a culture of $E$. coli NCIB 9270. Samples of blood from? vegans were kindly supplied by $\mathrm{Dr} F$. R. Ellis and $\mathrm{Mr} A$. Kurtha. The work was supported by a grant from the Wellcome Trust.

\section{REFERENCES}

Boxer, G. E., and Rickards, J. C. (1952), Arch, Biochem., 39, 7. Lindstrand, K. (1964). Nature (Lond.), 204, 188. , and Ståhlberg, K-G. (1963). Acta med. scand., 174, 665. Wilson, J., and Matthews, D. M (1966). Brit, med. J., 2,988 C

Linnell, J. C., Smith, A. D. M., Smith, C. L., Wilson, J., and Matthews D. M. (1968). Brit. med. J., 2, 215.

—-, Mackenzie, H. M., and Matthews, D. M. (1969). J. clini N Path., 22, 506.

Matthews, D. M. (1961). Lancet, 1, 1289.

$\longrightarrow$, Gunasegaram, R., and Linnell, J. C. (1967). J. clin. Path.u 20, 683.

Reizenstein, P. (1967). Blood, 29, 494.

Smith, E. Lester (1968). Plant Fds. hum. Nutr., 1, 7.

Ståhlberg, K-G. (1964). Scand. J. haemat., 1, 220. , (1967). Ibid, Suppl. 1.

_-, Radner, S., and Nordén, A. (1967). Ibid, 4, 312.

Toohey, J. I. and Barker, H. A. (1961). J. biol. Chem., 236, 560.

Wilson, J. and Matthews, D. M. (1966). Clin. Sci., 31, 1.

Wokes, F. and Picard, C. W. (1955). Amer. J. clin. Nutr., 3, 383.

Yagiri, Y. (1967). J. Vitaminol., 13, 228. 